Article

\title{
Pneumatic Performance Study of a High Pressure Ejection Device Based on Real Specific Energy and Specific Enthalpy
}

\author{
Jie Ren *, Fengbo Yang, Dawei Ma, Guigao Le and Jianlin Zhong \\ School of Mechanical Engineering, Nanjing University of Science and Technology, Nanjing 210094, \\ Jiangsu, China; E-Mails: yangfengbo.cool@163.com (F.Y.); ma-dawei@mail.njust.edu.cn (D.M.); \\ leguigao@njust.edu.cn (G.L.); njustzhongjianlin@163.com (J.Z.) \\ * Author to whom correspondence should be addressed; E-Mail: renjie@njust.edu.cn; \\ Tel.: +86-25-8431-5125.
}

Received: 8 May 2014; in revised form: 10 August 2014 / Accepted: 29 August 2014 /

Published: 3 September 2014

\begin{abstract}
In high-pressure dynamic thermodynamic processes, the pressure is much higher than the air critical pressure, and the temperature can deviate significantly from the Boyle temperature. In such situations, the thermo-physical properties and pneumatic performance can't be described accurately by the ideal gas law. This paper proposes an approach to evaluate the pneumatic performance of a high-pressure air catapult launch system, in which esidual functions are used to compensate the thermal physical property uncertainties of caused by real gas effects. Compared with the Nelson-Obert generalized compressibility charts, the precision of the improved virial equation of state is better than Soave-Redlich-Kwong (S-R-K) and Peng-Robinson (P-R) equations for high pressure air. In this paper, the improved virial equation of state is further used to establish a compressibility factor database which is applied to evaluate real gas effects. The specific residual thermodynamic energy and specific residual enthalpy of the high-pressure air are also derived using the modified corresponding state equation and improved virial equation of state which are truncated to the third virial coefficient. The pneumatic equations are established on the basis of the derived residual functions. The comparison of the numerical results shows that the real gas effects are strong, and the pneumatic performance analysis indicates that the real dynamic thermodynamic process is obviously different from the ideal one.
\end{abstract}

Keywords: thermodynamics; residual function; specific thermodynamic energy; specific enthalpy; high pressure air; compressibility factor 


\section{Introduction}

Compared to petroleum or electric systems, high pressure air has the advantages of no pollution, high power density, cheapness, reliable performance, recycling use, and being easy to maintain [1,2]. It has been applied to industrial automation, robot driving, compressed air powered vehicles, and even some special industries such as aeronautics, astronautics, and weapons design [3]. Typically, the charging and discharging performance and exergy analysis [4] are based on the equations of state. However, in high-pressure pneumatic dynamic thermodynamic processes, the pressure is much higher than the air critical pressure and the temperature can deviate significantly from the Boyle temperature. Since the thermodynamic properties based on the ideal gas are not authentic [5], the deviations in the mass and energy balances in thermodynamic process may not be acceptable. In this paper, the specific residual thermodynamic energy and specific residual enthalpy will be derived to compensate for the real gas effect.

Extensive studies have been conducted on high pressure pneumatic systems [6,7]. In these works, most of the thermodynamic property calculations are still based on the ideal gas assumption, although the specific thermodynamic energy and specific enthalpy of an ideal gas and a real gas can differ considerably under high pressure and low temperature conditions. In this study, we will also examine the deviation between the state variables computed by the real gas equation and ideal gas assumption.

Many semi-empirical formulas have been proposed to describe the properties of real gases, including the van der Waals equation [5], Redlich-Kwong (R-K) equation [8], Soave-Redlich-Kwong (S-R-K) equation [9], Benedict-Webb-Rubin (B-W-R) equation [10], and Peng-Robinson (P-R) equation [11,12]. With the development of the corresponding states principles, these equations are applicable to all kinds of gases within a certain pressure and temperature range. However, the precision of some of these equations is not satisfactory, or when calculating the thermodynamic variables by using high order nonlinear equations, like the S-R-K and P-R equations, it will lead to the extra problem of solving transcendental equations, which appearance is not desirable. The SAFT-type equation of state [13] is accurate enough to calculate thermodynamic variables of air, while the mathematical expression of the derived functions are complex.

\section{Derivation and Determination of Real Gas Equation of State for High Pressure Air}

According to the corresponding state law, the compressibility factor which indicates the deviation of real gas from ideal gas can be obtained from the table of corresponding states [14]:

$$
Z=\frac{P V_{m}}{R T}=\left(\frac{P_{c} V_{m c}}{R T_{c}}\right)\left(\frac{P_{r} V_{m r}}{T_{r}}\right)=Z_{c} * \varphi\left(P_{r}, T_{r}\right)
$$

The compressibility factor is a function of the corresponding pressure and temperature. The compressibility factor value of an ideal gas is 1 . For most gases, the $P-Z$ curves can be approximately considered to be linear when the pressure $P<0.5 P_{\mathrm{c}}$ or the temperature $T>5 T_{\mathrm{c}}$, and the compressibility factor $Z$ is nearly 1 . However, under high pressure conditions over $0.5 P_{\mathrm{c}}$, or low temperature conditions below $5 T_{\mathrm{c}}$, a reliable and simple real gas equation of state should be derived to fit the data.

The virial coefficients which are basic thermodynamic properties represent the non-ideal behavior of real gases. The importance of the virial coefficients lies in the fact that they are related directly to the interactions between molecules. The second virial coefficient represents the deviation behavior from 
ideality due to interactions between pairs of molecules, the third virial coefficient gives the effects of interactions of molecular triplets, and so on. The fourth and higher virial coefficients usually contribute little to the densities of gases and have relatively large uncertainties. Therefore, the accurate knowledge of the virial coefficients is of great significance. In order to improve the accuracy of calculation, most effort has been focused on obtaining the second $[15,16]$ and third virial coefficients $[17,18]$.

The volume serial form of the virial equation which is truncated to the third virial coefficient can be written as:

$$
\frac{P V_{m}}{R T}=1+\frac{B}{V_{m}}+\frac{C}{V_{m}^{2}}
$$

After the introduction of the critical pressure $P_{\mathrm{c}}$, the critical temperature $T_{\mathrm{c}}$, acentric factor $\omega$, and the extended corresponding states variable $\theta$ [19], the second and third virial coefficients of the corresponding state can be expressed as:

$$
\begin{gathered}
B_{r}=\frac{B P_{c}}{R T_{c}}=B_{r}^{0}\left(T_{r}\right)+\omega B_{r}^{1}\left(T_{r}\right)+\theta B_{r}^{2}\left(T_{r}\right) \\
C_{r}=\frac{C P_{c}^{2}}{\left(R T_{c}\right)^{2}}=C_{r}^{0}\left(T_{r}\right)+\omega C_{r}^{1}\left(T_{r}\right)+\theta C_{r}^{2}\left(T_{r}\right)
\end{gathered}
$$

where $B_{\mathrm{r}}^{0}\left(T_{\mathrm{r}}\right), C_{\mathrm{r}}^{0}\left(T_{\mathrm{r}}\right)$ are obtained by fitting data for small spherical molecules $(\omega=0) ; B_{\mathrm{r}}{ }^{1}\left(T_{\mathrm{r}}\right), C_{\mathrm{r}}{ }^{1}\left(T_{\mathrm{r}}\right)$ are obtained from data for larger, non-spherical, non-polar molecules $(\omega \neq 0) ; B_{\mathrm{r}}{ }^{2}\left(T_{\mathrm{r}}\right), C_{\mathrm{r}}^{2}\left(T_{\mathrm{r}}\right)$ are obtained from data for non-hydrogen bonding polar molecules; and $T_{\mathrm{r}}=T / T_{\mathrm{c}}$.

In this paper, the real gas is assumed to be air in chemical equilibrium [20]. The National Institute of Standards and Technology (NIST) provides a basic model of air [21], which consists of nitrogen, oxygen, and argon. These are non polar molecules, the $B_{\mathrm{r}}^{2}\left(T_{\mathrm{r}}\right)$, and $C_{\mathrm{r}}^{2}\left(T_{\mathrm{r}}\right)$ of dry air are 0 , and the critical parameters of air are: $T_{\mathrm{c}}=132.45 \mathrm{~K}, P_{\mathrm{c}}=3.77 \mathrm{MPa}$. The improved formulas of $B_{\mathrm{r}}{ }^{0}\left(T_{\mathrm{r}}\right), B_{\mathrm{r}}{ }^{1}\left(T_{\mathrm{r}}\right), C_{\mathrm{r}}{ }^{0}\left(T_{\mathrm{r}}\right)$, and $C_{\mathrm{r}}^{1}\left(T_{\mathrm{r}}\right)$ given by references [22-24] are:

$$
\begin{gathered}
B_{r}^{0}\left(T_{r}\right)=0.13356-\frac{0.30252}{T_{r}}-\frac{0.15668}{T_{r}^{2}}-\frac{0.00724}{T_{r}^{3}}-\frac{0.00022}{T_{r}^{8}} \\
B_{r}^{1}\left(T_{r}\right)=0.17404-\frac{0.15581}{T_{r}}+\frac{0.38183}{T_{r}^{2}}-\frac{0.44044}{T_{r}^{3}}-\frac{0.00541}{T_{r}^{8}} \\
C_{r}^{0}\left(T_{r}\right)=0.01407+\frac{0.02432}{T_{r}^{2.8}}-\frac{0.00313}{T_{r}^{10.5}} \\
C_{r}^{1}\left(T_{r}\right)=-0.02676+\frac{0.0177}{T_{r}^{2.8}}+\frac{0.04}{T_{r}^{3}}-\frac{0.003}{T_{r}^{6}}-\frac{0.00228}{T_{r}^{10.5}}
\end{gathered}
$$

The Peng-Robinson (P-R) equation, Equation (9), is an improvement on the van der Waals equation. It was proposed in 1976 [12,13]:

$$
p=\frac{R T}{v-b}-\frac{a(T)}{v(v+b)+b(v-b)}
$$


where $a(T)=0.42748\left(\frac{R^{2} T_{c}^{2}}{p_{c}}\right)\left[1+r\left(1-T_{r}^{0.5}\right)\right]^{2}, \quad r=0.48+1.574 w-0.176 w^{2}$, and $b=\frac{0.08664 R T_{c}}{p_{c}}$.

In the general high pressure pneumatic system, the pressure can reach $30 \mathrm{MPa}$, and the temperature range is about $250 \mathrm{~K}<T<400 \mathrm{~K}$. Compared with the Nelson-Obert's generalized compressibility chart [1], the P-R equation is more precise than the S-R-K equation in the compressibility factor calculation of air [1]. In this paper we calculate the compressibility factor, using improved virial and P-R equations. The results of some feature points are shown in Table 1. The data for air fitted in this paper are provided by the real properties database of National Institute of Standards and Technology (NIST) [25], as shown in Table 2.

Table 1. Compressibility factors of air using improved virial equation and P-R equation.

\begin{tabular}{|c|c|c|c|c|c|c|c|c|c|}
\hline \multirow{2}{*}{\multicolumn{3}{|c|}{ Compressibility Factors $Z$}} & \multicolumn{7}{|c|}{ Pressure [MPa] } \\
\hline & & & 0.101 & 1 & 6 & 12 & 19.43 & 25 & 30 \\
\hline \multirow{8}{*}{$\begin{array}{c}\text { Temperature } \\
{[\mathrm{K}]}\end{array}$} & \multirow{2}{*}{400} & virial & 1.0002 & 1.0019 & 1.0146 & 1.0358 & 1.0695 & 1.0986 & 1.1268 \\
\hline & & P-R & 1.0001 & 1.0007 & 1.0077 & 1.0231 & 1.0504 & 1.0755 & 1.1005 \\
\hline & \multirow{2}{*}{298} & virial & 0.9997 & 0.9969 & 0.9880 & 0.9922 & 1.0173 & 1.0470 & 1.0788 \\
\hline & & $\mathrm{P}-\mathrm{R}$ & 0.9994 & 0.9947 & 0.9772 & 0.9773 & 1 & 1.0275 & 1.0620 \\
\hline & \multirow{2}{*}{250} & virial & 0.9990 & 0.9907 & 0.9540 & 0.9351 & 0.9505 & 0.9832 & 1.0209 \\
\hline & & $\mathrm{P}-\mathrm{R}$ & 0.9987 & 0.9875 & 0.9392 & 0.9190 & 0.9382 & 0.9725 & 1.0170 \\
\hline & \multirow{2}{*}{200} & virial & 0.9976 & 0.9765 & 0.8687 & 0.7942 & 0.8133 & 0.8685 & 0.9278 \\
\hline & & P-R & 0.9970 & 0.9710 & 0.8476 & 0.7831 & 0.8157 & 0.8775 & 0.9465 \\
\hline
\end{tabular}

Table 2. Specific volume within different temperature and pressure.

\begin{tabular}{|c|c|c|c|c|c|c|c|c|c|c|}
\hline \multirow{2}{*}{\multicolumn{2}{|c|}{$\begin{array}{c}\text { Specific volume } \\
{\left[\mathrm{m}^{3} / \mathrm{kg}\right]}\end{array}$}} & \multicolumn{9}{|c|}{ Pressure [MPa] } \\
\hline & & 0.1 & 0.5 & 1 & 6 & 10 & 15 & 20 & 25 & 30 \\
\hline \multirow{6}{*}{$\begin{array}{c}\text { Temperature } \\
{[\mathrm{K}]}\end{array}$} & 100 & 0.281 & 0.0509 & 0.00130 & 0.00126 & 0.00125 & 0.00122 & 0.00120 & 0.00119 & 0.00117 \\
\hline & 140 & 0.399 & 0.0773 & 0.0370 & 0.00222 & 0.00174 & 0.00158 & 0.00150 & 0.00144 & 0.00139 \\
\hline & 200 & 0.537 & 0.114 & 0.0561 & 0.00833 & 0.00467 & 0.00309 & 0.00245 & 0.00214 & 0.00195 \\
\hline & 260 & 0.746 & 0.149 & 0.0741 & 0.0120 & 0.00713 & 0.00478 & 0.00368 & 0.00307 & 0.00269 \\
\hline & 300 & 0.861 & 0.172 & 0.0859 & 0.0142 & 0.00855 & 0.00578 & 0.00446 & 0.00368 & 0.00318 \\
\hline & 400 & 0.148 & 0.230 & 0.115 & 0.0194 & 0.0118 & 0.00806 & 0.00620 & 0.00509 & 0.00437 \\
\hline
\end{tabular}

The error results when calculating the compressibility factor within the pressure range of $0.101325 \mathrm{MPa}<P<30 \mathrm{MPa}$ at the temperatures of $400 \mathrm{~K}, 300 \mathrm{~K}$ and $260 \mathrm{~K}$, respectively, are shown as Table 3. From Table 3, it is found that the precision of improved virial equation is better than P-R equation compared to the NIST database.

Compared with the P-R equation, the precision of the improved virial equation is better. The precision can meet the requirements of general engineering computation. Therefore, the improved virial equation is adopted to calculate compressibility factor, and in this paper the thermodynamic variables will be derived based on the improved virial equation of state. 
Table 3. Error of compressibility factors for air using improved virial equation and P-R equation respectively.

\begin{tabular}{|c|c|c|c|c|c|}
\hline \multirow[b]{2}{*}{$\begin{array}{c}\text { Temperature } \\
{[\mathrm{K}]}\end{array}$} & \multirow[b]{2}{*}{$\begin{array}{c}\text { Pressure range } \\
\text { [MPa] }\end{array}$} & \multicolumn{2}{|c|}{ P-R equation } & \multicolumn{2}{|c|}{ Improved virial equation } \\
\hline & & $\begin{array}{c}\text { Maximum } \\
\text { absolute } \\
\text { error }\end{array}$ & $\begin{array}{c}\text { Maximum } \\
\text { relative } \\
\text { error }\end{array}$ & $\begin{array}{c}\text { Maximum } \\
\text { absolute } \\
\text { error }\end{array}$ & $\begin{array}{c}\text { Maximum } \\
\text { relative } \\
\text { error }\end{array}$ \\
\hline 400 & $0.101325 \sim 30$ & 0.0415 & $3.63 \%$ & 0.0152 & $1.33 \%$ \\
\hline 300 & $0.101325 \sim 30$ & 0.0467 & $4.21 \%$ & 0.0274 & $2.47 \%$ \\
\hline 260 & $0.101325 \sim 30$ & 0.0555 & $5.13 \%$ & 0.0457 & $4.23 \%$ \\
\hline
\end{tabular}

Figure 1 shows the relationship between the pressure and density of high pressure air under different temperature conditions using different equations of state. It is found that the improved virial equation shows good agreement with the NIST data.

Figure 1. Comparison of real gas equations of state ( $T=400 \mathrm{~K}, T=300 \mathrm{~K}, T=260 \mathrm{~K})$.

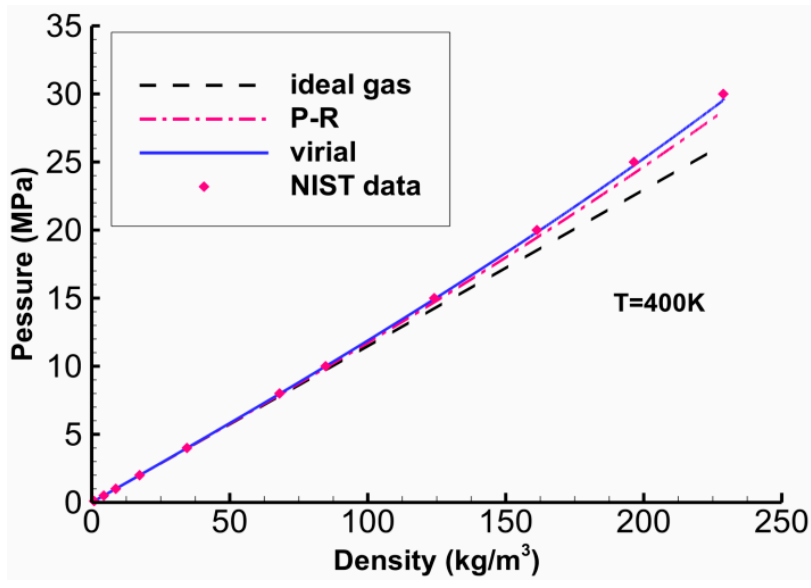

(a)

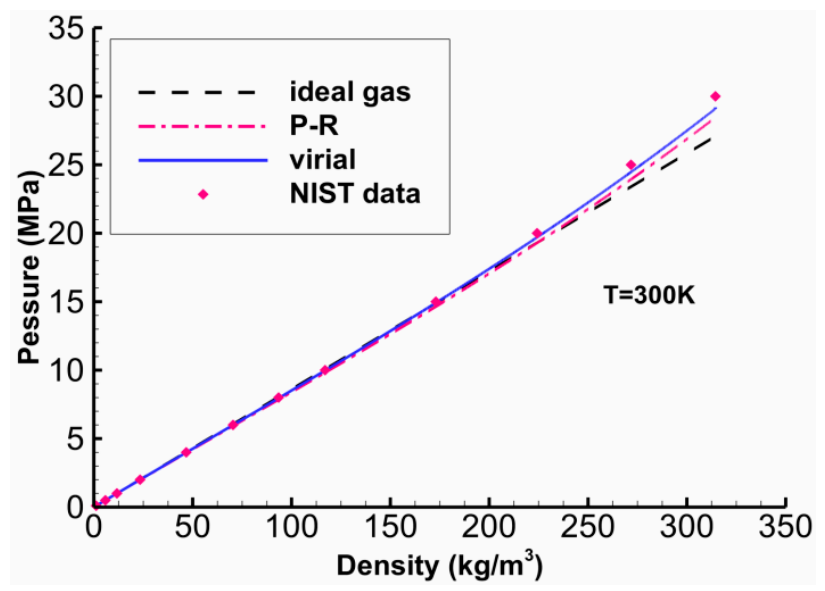

(b)

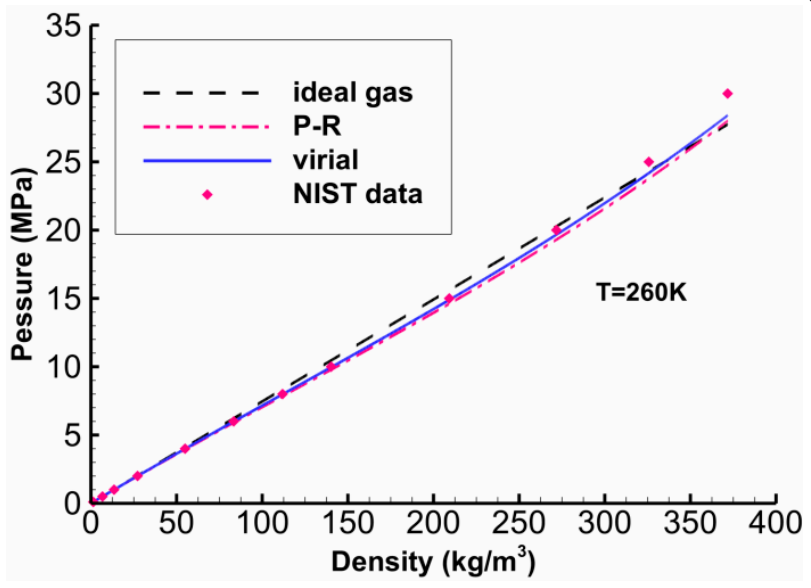

(c)

\section{Modeling Thermodynamic Variables}

The dynamic thermodynamic analysis under high pressure conditions such as mass flow rate, charging and discharging processes, and exergy analysis in the pneumatic system are of particular interest in many applications. Therefore, to investigate the thermal behaviors in thermodynamic 
processes, which can be used to predict the gas pressure, temperature and flow rate, it is essential to derive the thermodynamic variables on the basis of real gas equation states. In this section, the real analytical expressions of specific thermodynamic energy and specific enthalpy will be derived for high pressure air.

\subsection{Residual Functions}

In the calculation of real thermodynamic property variables, the ideal value can be calculated first, then the residual function will subtracted from the ideal value. The definition of residual function can be expressed as:

$$
F_{\text {re }}=F^{*}-F
$$

where $F_{\text {re }}$ denotes the residual of a arbitrary extensive properties or specific properties, that is, the difference between the properties of ideal gas and real gas, $F^{*}$ denotes the properties of ideal gas, and $F$ denotes the properties of real gas.

The differential form of specific thermodynamic energy for real gas is:

$$
d u=c_{v} d T-\left[p-T\left(\frac{\partial P}{\partial T}\right) v\right] d v
$$

We have:

$$
\left(\frac{\partial u}{\partial v}\right)_{T}=T\left(\frac{\partial p}{\partial T}\right)_{v}-p
$$

The residual specific thermodynamic energy for a real gas is obtained by integrating the equation above from $v^{*}=\infty$ (ideal gas state) to $v$ (real gas state) along the isotherm:

$$
u_{r}=\int_{\infty}^{v}\left[p-T\left(\frac{\partial p}{\partial T}\right)\right] d v=-T^{2} \int_{\infty}^{v}\left[\frac{\partial(p / T)}{\partial T}\right]_{v} d v
$$

The specific enthalpy for a real gas is defined as:

$$
h=u+p v
$$

and the specific enthalpy for an ideal gas can be expressed by:

$$
h^{*}=u^{*}+R_{g} T
$$

Combination of the above three equations will lead to:

$$
h_{r}=-T^{2} \int_{\infty}^{v}\left[\frac{\partial(p / T)}{\partial T}\right]_{v} d v-p v+R_{g} T
$$

\subsection{Thermodynamic Variables of Ideal Gas}

The specific thermodynamic energy and specific enthalpy of ideal gas can be written as:

$$
u^{*}(T)=\int_{T_{0}}^{T} c_{V}^{*} d T+u_{0}
$$




$$
h^{*}(T)=\int_{T_{0}}^{T}\left(c_{V}^{*}+R_{g}\right) d T+h_{0}
$$

For ideal gas, isochoric heat capacity can be expressed by:

$$
c_{V}^{*}=R_{g}\left((\alpha-1)+\beta T+\gamma T^{2}+\delta T^{3}+\varepsilon T^{4}\right)
$$

where $\alpha=3.653, \beta=-1.337 \times 10^{-3}, \gamma=3.294 \times 10^{-6}, \delta=-1.913 \times 10^{-9}$, and $\varepsilon=0.2763 \times 10^{12}$.

\subsection{Thermodynamic Variables of Real Gas}

Based on the improved virial equation and substituting Equations (13), (16), (17) and (18) into Equation (10), respectively, the analytical expressions of specific thermodynamic energy and specific enthalpy for real gas are obtained as follows:

$$
\begin{aligned}
& u=\int_{T_{0}}^{T}\left(R_{g}\left((\alpha-1)+\beta T+\gamma T^{2}+\delta T^{3}+\varepsilon T^{4}\right)\right) d T+u_{0} \\
& -\left[\frac { R R _ { g } T _ { c } } { p _ { c } V _ { m } } \left(0.30252 T_{c}+\frac{0.31336 T_{c}^{2}}{T}+\frac{0.02172 T_{c}^{3}}{T^{2}}+\frac{0.00176 T_{c}^{8}}{T^{7}}\right.\right. \\
& \left.+w\left(0.15581 T_{c}-\frac{0.763662 T_{c}^{2}}{T}+\frac{1.32132 T_{c}^{3}}{T^{2}}+\frac{0.04328 T_{c}^{8}}{T^{7}}\right)\right) \\
& \left.+\frac{R^{2} R_{g} T_{c}^{2}}{2 p_{c}^{2} V_{m}^{2}}\left(-\frac{0.068096 T_{c}^{2.8}}{T^{1.8}}+\frac{0.032865 T_{c}^{10.5}}{T^{9.5}}+w\left(-\frac{0.04956 T_{c}^{2.8}}{T^{1.8}}-\frac{0.12 T_{c}^{3}}{T^{2}}+\frac{0.018 T_{c}^{6}}{T^{5}}+\frac{0.02394 T_{c}^{10.5}}{T^{9.5}}\right)\right)\right] \\
& h=\int_{T_{0}}^{T}\left(R_{g}\left(\alpha+\beta T+\gamma T^{2}+\delta T^{3}+\varepsilon T^{4}\right)\right) d T+h_{0}- \\
& -\left[\frac { R R _ { g } T _ { c } } { p _ { c } V _ { m } } \left(0.30252 T_{c}+\frac{0.31336 T_{c}^{2}}{T}+\frac{0.02172 T_{c}^{3}}{T^{2}}+\frac{0.00176 T_{c}^{8}}{T^{7}}\right.\right. \\
& \left.+w\left(0.15581 T_{c}-\frac{0.763662 T_{c}^{2}}{T}+\frac{1.32132 T_{c}^{3}}{T^{2}}+\frac{0.04328 T_{c}^{8}}{T^{7}}\right)\right) \\
& \left.+\frac{R^{2} R_{g} T_{c}^{2}}{2 p_{c}^{2} V_{m}^{2}}\left(-\frac{0.068096 T_{c}^{2.8}}{T^{1.8}}+\frac{0.032865 T_{c}^{10.5}}{T^{9.5}}+w\left(-\frac{0.04956 T_{c}^{2.8}}{T^{1.8}}-\frac{0.12 T_{c}^{3}}{T^{2}}+\frac{0.018 T_{c}^{6}}{T^{5}}+\frac{0.02394 T_{c}^{10.5}}{T^{9.5}}\right)\right)\right] \\
& +R_{g} T\left(\frac{R T_{c}\left(B_{r}^{0}\left(T_{r}\right)+\omega B_{r}^{1}\left(T_{r}\right)+\theta B_{r}^{2}\left(T_{r}\right)\right)}{v M P_{c}}+\frac{\left(R T_{c}\right)^{2}\left(C_{r}^{0}\left(T_{r}\right)+\omega C_{r}^{1}\left(T_{r}\right)+\theta C_{r}^{2}\left(T_{r}\right)\right)}{v^{2} M^{2} P_{c}^{2}}\right)
\end{aligned}
$$

\section{Modeling of Pneumatic Catapult}

Figure 2 shows the working principle of a high pressure pneumatic catapult, and Figure 3 is the schematic diagram of the lifting ejection mechanism which is a schematic structural diagram of the three-step piston cylinder in Figure 1 and component 6 in Figure 4. There $d_{a}$ is the diameter of the piston rod, $d_{c}$ is the diameter of piston, $S_{a}$ is an effective thrust area of the third stage cylinder, $L_{b}$ represents the second cylinder, and $L_{c}$ represents the third stage cylinder. 
Figure 2. High pressure gas supply system.

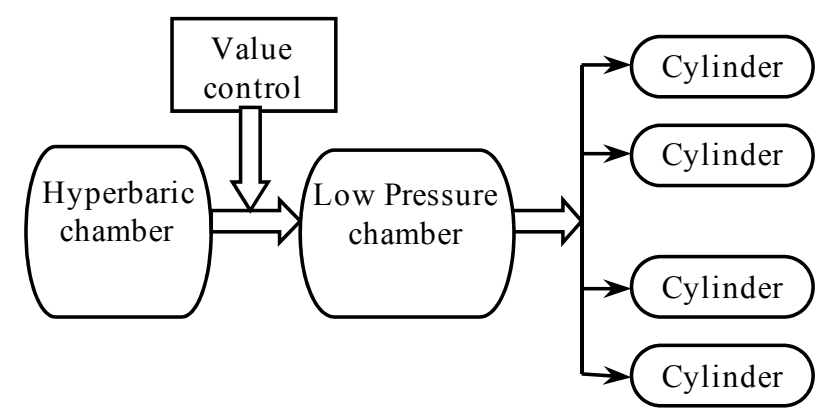

The working principle of the lifting ejection system can be described as follows: as the launching process starts, the controlled valve immediately responds and opens, and high-pressure gas flows into the low pressure chamber; the second stage of the cylinder pushes the piston to move forward, then the first stage of the cylinder pushes the piston to move forward after the second stage moves to the end; missile moves with lifting beam, then lifting beam collides with the buffer, and missile flies out of the launch tube.

In the course of the pneumatic ejection, taking the subsonic and sonic flow into account, mass flow equation can be written as:

$$
G_{c}= \begin{cases}\mu_{x} \frac{p_{1} A}{\left.\sqrt{R_{g} T_{1}} \sqrt{\frac{2 k}{k-1}\left[\left(\frac{p_{2}}{p_{1}}\right)^{\frac{2}{k}}-\left(\frac{p_{2}}{p_{1}}\right)^{\frac{k+1}{k}}\right.}\right],} & \left(\frac{2}{k+1}\right)^{\frac{k}{k-1}}<\frac{p_{2}}{p_{1}}<1 \\ \mu_{x} \sqrt{k}\left(\frac{2}{k+1}\right)^{\frac{k+1}{2(k-1)}} p_{1} A / \sqrt{R_{g} T_{1}}, & \frac{p_{2}}{p_{1}} \leq\left(\frac{2}{k+1}\right)^{\frac{k}{k-1}}\end{cases}
$$

where subscripts 1 and 2 indicate high-pressure chamber and low pressure chamber respectively, $\mu_{\mathrm{x}}$ is flow correction factor, $A$ denotes the equivalent cross-sectional area of orifice, and $k$ denotes the adiabatic index.

According to mass and energy conservation laws, and the flow equation in the high-pressure chamber and low pressure chamber, the following relations can be established:

$$
\left\{\begin{array}{l}
\frac{d}{d t}\left(\rho_{1} V_{1}\right)=-Q_{\mathrm{m}} \\
\frac{d}{d t}\left(\rho_{1} V_{1} u_{1}\right)=-Q_{\mathrm{m}} h_{1} \\
p_{1}=\frac{R T_{1}}{V_{\mathrm{m} 1}}\left(1+\frac{B_{1}}{V_{\mathrm{m} 1}}+\frac{C_{1}}{V_{\mathrm{m} 1}^{2}}\right) \\
\frac{d m_{2}}{d t}=Q_{\mathrm{m}} \\
\frac{d}{d t}\left(m_{2} u_{2}\right)=Q_{\mathrm{m}} h_{1}-n s_{\mathrm{t}} p_{2} \frac{d l}{d t} \\
m_{\mathrm{e}} \frac{d v_{2}}{d t}=n\left(p_{2}-p_{\mathrm{a}}\right) s_{\mathrm{t}}-1.2 m_{\mathrm{e}} g \sin (\alpha) \\
p_{2}=\frac{R T_{2}}{V_{\mathrm{m} 2}}\left(1+\frac{B_{2}}{V_{\mathrm{m} 2}}+\frac{C_{2}}{V_{\mathrm{m} 2}^{2}}\right)
\end{array}\right.
$$


where $m_{\mathrm{e}}$ is the quality of missile, $P_{\mathrm{a}}$ denotes the atmospheric pressure, $g$ is the acceleration of gravity, $v_{2}$ is the speed of missile, and $s_{\mathrm{t}}$ denotes effective thrust area.

Let $X_{1}=\rho_{1}, X_{2}=T_{1}, X_{3}=m_{2}, X_{4}=T_{2}, X_{5}=l, X_{6}=v_{2}$, the closed pneumatic equations can be established as:

$$
\begin{aligned}
& \left\{\begin{array}{l}
\dot{X}_{1}=-G_{c} / V_{1} \\
\dot{X}_{2}=\frac{\frac{-G_{c}}{V_{1}}\left\{\left[\frac{R_{g} R T_{c}}{p_{c} V_{m 1}} D_{111}\left(1+\frac{M_{0}}{X_{1} V_{m 1}}\right)+\frac{R_{g} R^{2} T_{c}^{2}}{p_{c}^{2} V_{m 1}^{2}} D_{121}\left(\frac{1}{2}+\frac{M_{0}}{X_{1} V_{m 1}}\right)\right]-\int_{298.15}^{X_{2}} c_{V}^{*} d T-u_{0}\right\}-G_{c}\left(h_{1}^{*}-h_{1 r}\right) / V_{1}}{X_{1}\left[c_{V}^{*}-\frac{R_{g} R T_{c}}{p_{c} V_{m 1}} D_{211}-\frac{R_{g} R^{2} T_{c}^{2}}{2 p_{c}^{2} V_{m 1}^{2}} D_{221}\right]}
\end{array}\right. \\
& \dot{X}_{3}=G_{c} \\
& \dot{X}_{4}=\left\{\left[\int_{298.15}^{X_{4}} c_{V}^{*} d T+e-\left(\frac{R_{g} R T_{c}}{p_{c} V_{m 2}} D_{112}+\frac{R_{g} R^{2} T_{c}^{2}}{2 p_{c}^{2} V_{m 2}^{2}} D_{122}\right)-\frac{R_{g} R T_{c} M_{0}\left(V_{0}+n s_{0} X_{5}\right)}{p_{c} V_{m 2}^{2} X_{3}}\left(1+\frac{R T_{c}}{p_{c} V_{m 2}}\right)-\left(h_{1}^{*}-h_{1 r}\right)\right] G_{c}\right. \\
& \left.+n s_{0} p_{2} X_{6}+\frac{R_{g} R T_{c} M_{0} n s_{0} X_{6}}{p_{c} V_{m 2}^{2}}\left(1+\frac{R T_{c}}{p_{c} V_{m 2}}\right)\right\} /\left[-X_{3} c_{V}^{*}+\frac{X_{3} R_{g} R T_{c}}{p_{c} V_{m 2}} D_{212}+\frac{X_{3} R_{g} R^{2} T_{c}^{2}}{2 p_{c}^{2} V_{m 2}^{2}} D_{222}\right] \\
& \dot{X}_{5}=X_{6} \\
& \dot{X}_{6}=\left\{\begin{array}{lc}
\frac{n\left(p_{2}-p_{0}\right) s_{0}-1.2 M_{0} g \sin (\alpha)}{M_{0}}, & p_{2} \geq p_{0}+1.2 \frac{M_{0} g \sin (\alpha)}{n s_{0}} \\
0, & p_{2}<p_{0}+1.2 \frac{M_{0} g \sin (\alpha)}{n s_{0}}
\end{array}\right. \\
& D_{211}=\frac{-0.31336 T_{c}^{2}}{X_{2}^{2}}-\frac{0.04344 T_{c}^{3}}{X_{2}^{3}}-\frac{0.01232 T_{c}^{8}}{X_{2}^{8}}+\omega\left(\frac{0.763662 T_{c}^{2}}{X_{2}^{2}}-\frac{2.64264 T_{c}^{3}}{X_{2}^{3}}-\frac{0.30296 T_{c}^{8}}{X_{2}^{8}}\right) \\
& D_{221}=\frac{0.1225728 T_{c}^{2.8}}{X_{2}^{2.8}}-\frac{0.3122175 T_{c}^{10.5}}{X_{2}^{10.5}}+\omega\left(\frac{0.089208 T_{c}^{2.8}}{X_{2}^{2.8}}+\frac{0.24 T_{c}^{3}}{X_{2}^{3}}-\frac{0.09 T_{c}^{6}}{X_{2}^{6}}-\frac{0.22743 T_{c}^{10.5}}{X_{2}^{10.5}}\right) \\
& \mathrm{D}_{112}=0.30252 T_{c}+\frac{0.31336 T_{c}^{2}}{X_{4}}+\frac{0.02172 T_{c}^{3}}{X_{4}^{2}}+\frac{0.00176 T_{c}^{8}}{X_{4}^{7}} \\
& +\omega\left(0.15581 T_{c}-\frac{0.763662 T_{c}^{2}}{X_{4}}+\frac{1.32132 T_{c}^{3}}{X_{4}^{2}}+\frac{0.04328 T_{c}^{8}}{X_{4}^{7}}\right) \\
& \mathrm{D}_{122}=-\frac{0.068096 T_{c}^{2.8}}{X_{4}^{1.8}}+\frac{0.032865 T_{c}^{10.5}}{X_{4}^{9.5}}+\omega\left(-\frac{0.04956 T_{c}^{2.8}}{X_{4}^{1.8}}-\frac{0.12 T_{c}^{3}}{X_{4}^{2}}+\frac{0.018 T_{c}^{6}}{X_{4}^{5}}+\frac{0.02394 T_{c}^{10.5}}{X_{4}^{9.5}}\right) \\
& D_{212}=\frac{-0.31336 T_{c}^{2}}{X_{4}^{2}}-\frac{0.04344 T_{c}^{3}}{X_{4}^{3}}-\frac{0.01232 T_{c}^{8}}{X_{4}^{8}}+\omega\left(\frac{0.763662 T_{c}^{2}}{X_{4}^{2}}-\frac{2.64264 T_{c}^{3}}{X_{4}^{3}}-\frac{0.30296 T_{c}^{8}}{X_{4}^{8}}\right) \\
& D_{222}=\frac{0.1225728 T_{c}^{2.8}}{X_{4}^{2.8}}-\frac{0.3122175 T_{c}^{10.5}}{X_{4}^{10.5}}+\omega\left(\frac{0.089208 T_{c}^{2.8}}{X_{4}^{2.8}}+\frac{0.24 T_{c}^{3}}{X_{4}^{3}}-\frac{0.09 T_{c}^{6}}{X_{4}^{6}}-\frac{0.22743 T_{c}^{10.5}}{X_{4}^{10.5}}\right)
\end{aligned}
$$


Figure 3. Three-step piston cylinder simplified diagram.

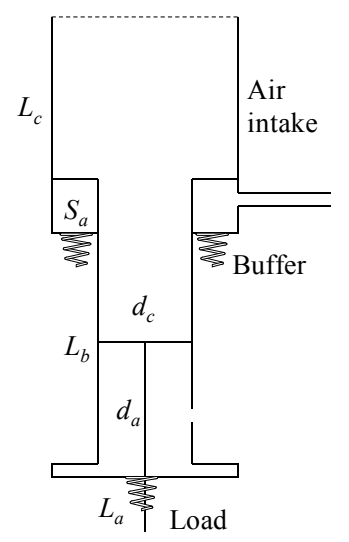

Figure 4. Lifting ejection mechanism sketch.

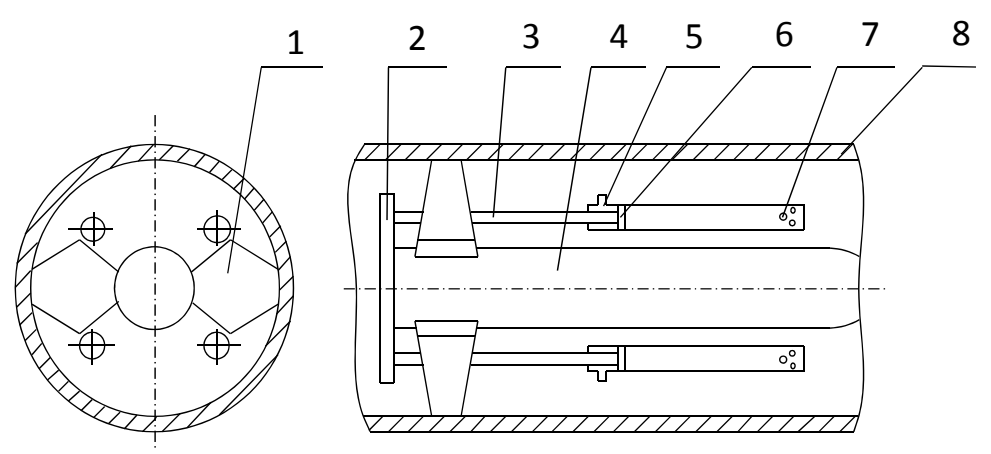

1-folding wing, 2-lifting beam, 3-lifting pole, 4-missile, 5-gas inlet, 6-three-step piston cylinder, 7-exhaust orifice, 8-launch tube.

\section{Simulation Analysis of the Pneumatic Ejection}

The system parameters of above mathematical model and thermo-physical parameters are as shown in Table 4. The five-step four-order Runge-Kutta method is used to calculate the pneumatic ejection process based on ideal gas equation and real gas equation respectively. The basic idea of five-step four-order Runge-Kutta method is expressed as:

$$
\left\{\begin{array}{l}
k_{i 1}=f_{i}\left(t_{j}, X_{1 j}, \ldots, X_{6 j}\right) \\
k_{i 2}=f_{i}\left(t_{j}+\frac{t_{0}}{2}, X_{1 j}+\frac{t_{0} k_{11}}{2}, \ldots, X_{6 j}+\frac{t_{0} k_{61}}{2}\right) \\
k_{i 3}=f_{i}\left(t_{j}+\frac{t_{0}}{2}, X_{1 j}+\frac{t_{0} k_{12}}{2}, \ldots, X_{6 j}+\frac{t_{0} k_{62}}{2}\right), \quad i=1,2, \ldots, 6 \\
k_{i 4}=f_{i}\left(t_{j}+t_{0}, X_{1 j}+t_{0} k_{13}, \ldots, X_{6 j}+t_{0} k_{63}\right) \\
X_{i, j+1}=X_{i, j}+t_{0}\left(\frac{k_{i 1}}{6}+\frac{k_{i 2}}{3}+\frac{k_{i 3}}{3}+\frac{k_{i 4}}{6}\right)
\end{array}\right.
$$

where $t_{0}$ indicates time step, and $j$ represents the current time step. 
Table 4. System parameters and thermal physical parameters of dry air.

\begin{tabular}{ll}
\hline Parameters & Values \\
\hline Universal gas constant $R[\mathrm{~J} / \mathrm{mol} \cdot \mathrm{K}]$ & 8.3145 \\
Critical temperature $T_{\mathrm{c}}[\mathrm{K}]$ & 132.45 \\
Critical pressure $P_{\mathrm{c}}[\mathrm{MPa}]$ & 3.77 \\
Acentric Factor $\omega$ & 0.031 \\
Gas constant of air $R_{\mathrm{g}}[\mathrm{J} / \mathrm{kg} \cdot \mathrm{K}]$ & 287.0 \\
Molar mass $M$ [g/mol] & 28.97 \\
Flow correction factor $\mu_{\mathrm{x}}$ & 0.95 \\
Cross-sectional area valve control $A\left[\mathrm{~m}^{2}\right]$ & 0.0123 \\
Adiabatic index $k$ & 1.4 \\
Initial gas density of " 1 " $\rho_{1}\left[\mathrm{~kg} / \mathrm{m}^{3}\right]$ & 360 \\
Initial gas temperature of " 1 " $T_{1}[\mathrm{~K}]$ & 300 \\
Volume of "1" $V_{1}\left[\mathrm{~m}^{3}\right]$ & 1.8 \\
Initial gas mass of " 2 " $m_{2}[\mathrm{~kg}]$ & 26 \\
Initial gas temperature of " 2 " $T_{2}[\mathrm{~K}]$ & 300 \\
The number three piston cylinder $n$ & 4 \\
Effective thrust area of cylinder $S_{0}\left[\mathrm{~m}^{2}\right]$ & 0.0235 \\
Mass of missile $M_{0}[\mathrm{~kg}]$ & 24,000 \\
Launch angle $\alpha[\mathrm{deg}]$ & 90 \\
Initial volume of "2" $V_{0}\left[\mathrm{~m}^{3}\right]$ & 0.7 \\
\hline
\end{tabular}

\subsection{Comparative Analysis of Dynamic Thermodynamic Processes}

Figures 5-9 show the dynamic variation comparison of the main thermodynamic variables in the ejection process. When air flows from high pressure chamber to low pressure chamber in the throttling process, the temperature will change with the pressure drop. The throttling process is assumed to be an isenthalpic process. From Figure 5, we can see that the high pressure air flows into the low pressure chamber, the temperature in the high pressure chamber decreases all the time. In the initial stage, the temperature of the low-pressure chamber increases immediately, and then decreases gradually when the missile moves upward. Also, from Figure 5, we can see that the real temperature in the high chamber decreases faster than the ideal temperature before $0.2 \mathrm{~s}$. This can be explained from the following two aspects: firstly, Figure 9 shows that the gas mass based on real gas and ideal gas flowing into the low pressure chamber are nearly equal; secondly, Figure 7 shows that the residual enthalpy in high pressure chamber is positive, the real enthalpy of high pressure air is obviously less than ideal one. As a result, the real temperature in the high chamber decreases faster before $0.2 \mathrm{~s}$.

From Figure 5, we know the temperature in the low pressure chamber increases immediately, and then decreases slowly when the missile moves upward. It shows that the real temperature of gas in the low pressure is always lower than ideal one, which indicates that the real gas effects decelerates the temperature increasing rates in the low pressure chamber in the early stage, and accelerates the rate of decrease of the temperature with the missile moving upward.

Figure 6 shows the pressure variations in the high pressure chamber and low pressure chamber. We can see that the hyperbaric always deflates, and the pressure continues to decay. High pressure air flows into low pressure chamber that the pressure in the low pressure chamber increases before the missile 
starts to move, and decreases as the missile moves upward. The real pressure decay rate is greater than the ideal decay rate. This is due to the following facts: firstly, from the analysis above, we know that the real temperature in the high chamber decreases faster than the ideal one; secondly, the real gas mass flowing out of high pressure chamber is nearly the same as the ideal mass. The first factor is the dominant factor which makes the phenomenon happened. The ideal value of temperature in the hyperbaric pressure chamber is much greater than the actual value which is similar to low pressure chamber.

Figure 5. Temperature contrast curve.

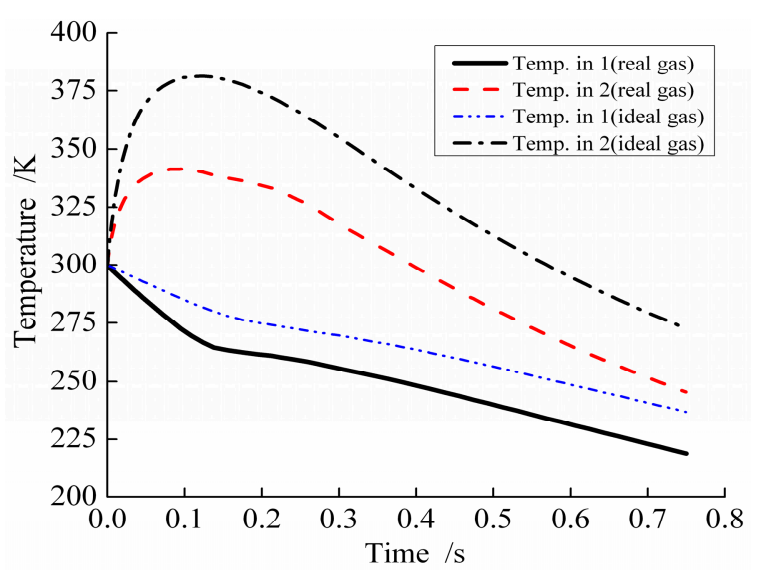

Figure 6. Pressure contrast curve.

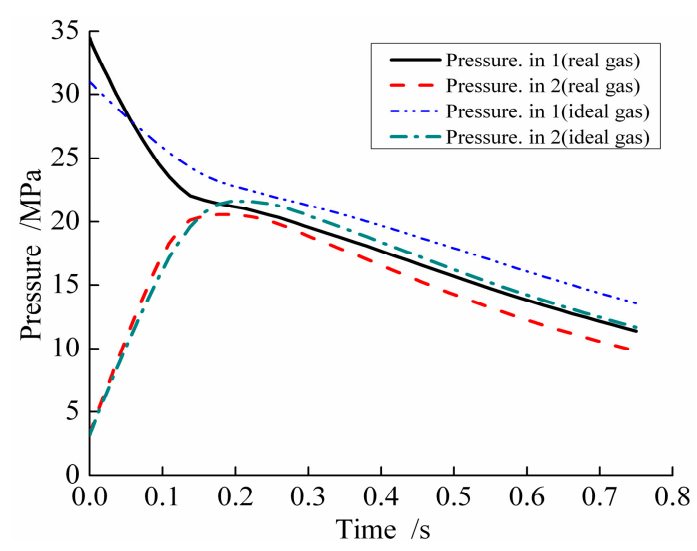

From Figure 7, we can see that the specific enthalpy in the hyperbaric chamber increases first and then decreases slightly, while the specific enthalpy in the low pressure chamber rises rapidly, and then increases slowly. When the pressure getting higher, the distance between molecules becomes smaller and the intermolecular interaction becomes stronger, as a result, the specific enthalpy gets larger and it deviates more from the ideal gas state. While the temperature increases, the situation is totally different: longer distance between molecules makes the intermolecular forces smaller, so the specific enthalpy gets smaller and it is more close to the ideal gas state. For the high pressure chamber, with gas flowing into low pressure chamber, pressure decays, and temperature decreases rapidly. Figure 7 shows the specific enthalpy in the high pressure chamber drops slightly, which indicates that the pressure drop is the main factor. For the low pressure chamber, the pressure and temperature increase first, and decrease with the gas pushing the missile upwards. Figure 7 shows the specific enthalpy in the low pressure chamber increases rapidly first and then increases slightly, which indicates that the pressure increase is the main factor in the early stage, and the temperature decrease is the main factor in the later round. 
Figure 7. The residual enthalpy curve.

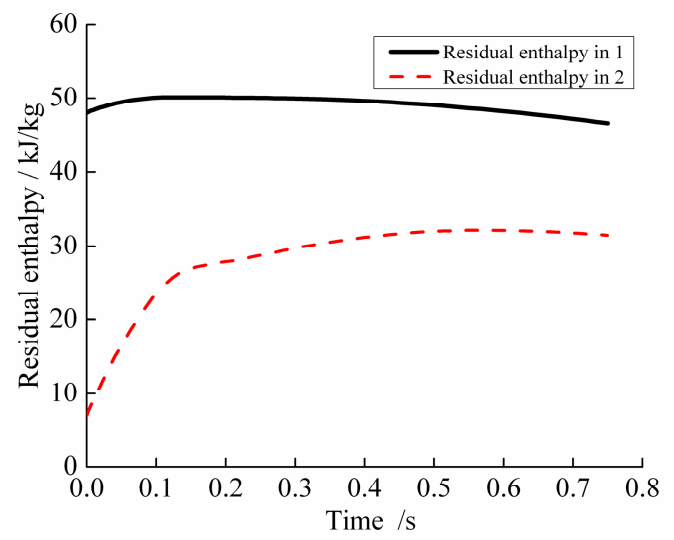

Figure 8 shows the dynamic variation of pressure compression factors in the hyperbaric chamber and low pressure chamber. The maximum compression factor reaches 1.1013 and 1.0486 , respectively. From Equation (1) we can see that the compressibility factor is determined by temperature and pressure. As shown in Figure 5 and Figure 8, the compressibility factor variation law is similar to the pressure one which indicates that the pressure dominates the changing regulation of the compression factor.

Figure 8. The compressibility factor curve.

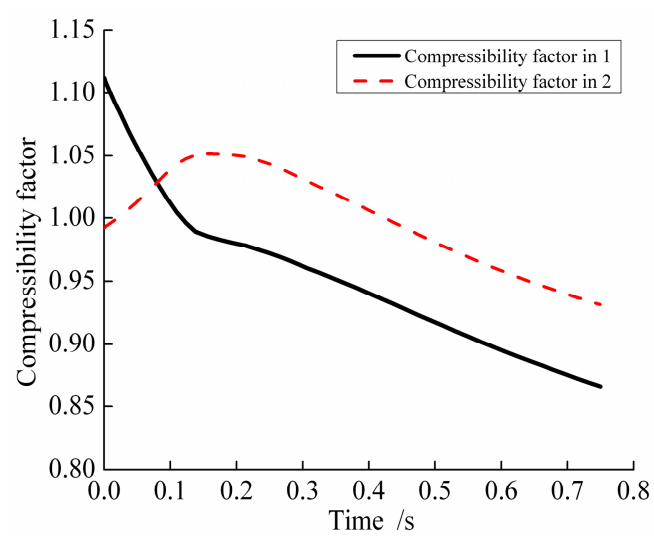

Figure 9 shows the mass flow rate declines rapidly at first, and then rises slowly. It is also noticed in Figure 9 that the real gas effect accelerates the rate of decrease of the mass flow in the early stage, and decelerates the rates of increase in the later period.

Figure 9. The mass flow rate curve.

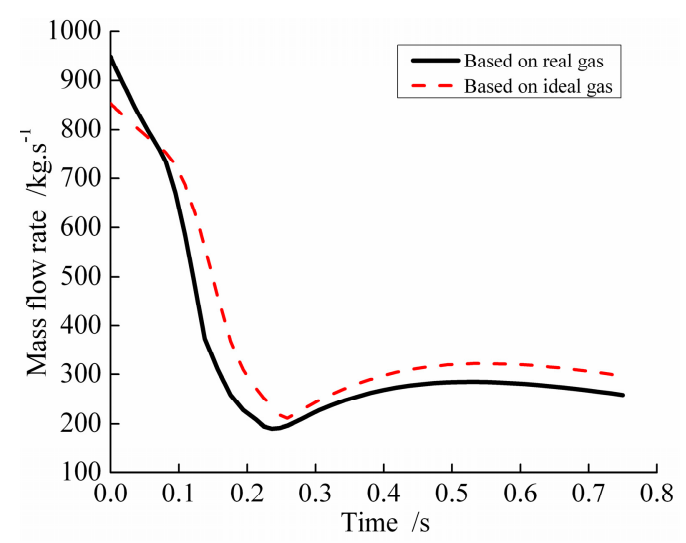




\subsection{Ejection Performance Evaluation Variables}

With the missile overload consistent with the pressure in the low pressure chamber, agreement between the acceleration of missile and pressure in the low pressure chamber are fairly satisfied, regardless of whether the correspondence relationship is based on a real gas or based on an ideal gas, just as shown in Figures 5 and 9.

The missile speed is a linear function of acceleration, and the missile stroke is a quadratic function of acceleration, while the ejection time is less than $1 \mathrm{~s}$. It is shown that the velocity of missile based on an ideal gas is significantly greater than that based on a real gas, and missile stroke based on an ideal gas is slightly larger than that based on a real gas shown in Figures 10-12.

Figure 10. The acceleration curves.

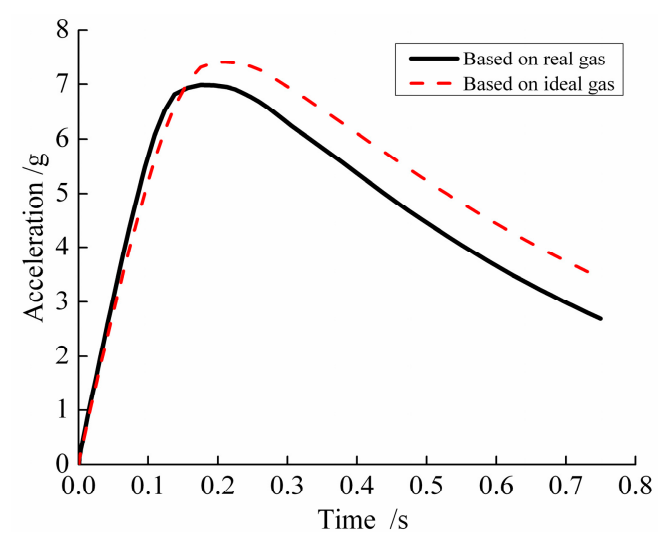

Figure 11. The velocity curve.

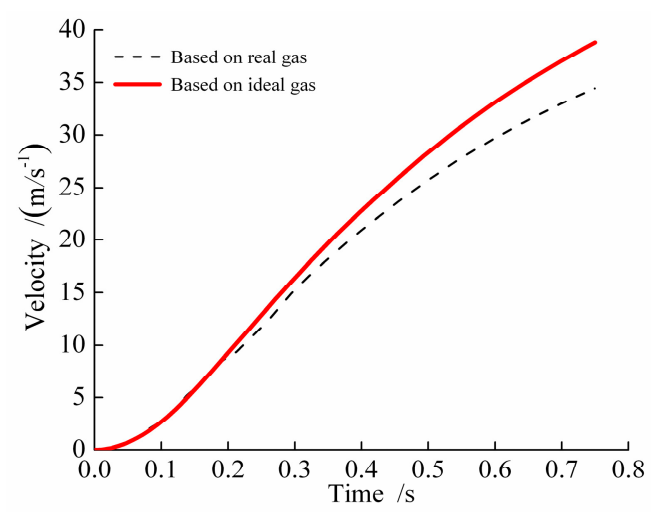

Figure 12. The displacement curve.

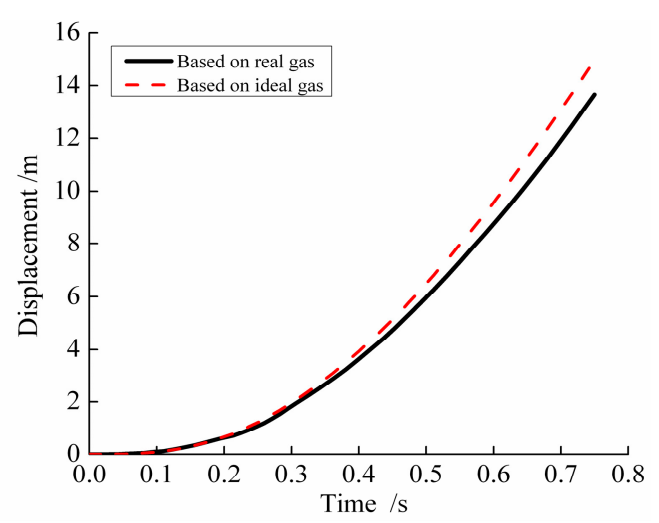




\section{Conclusions}

In this paper, the improved virial equation of state is used to describe the thermodynamic properties of high pressure air by fitting the NIST data. The compressibility factor is utilized to evaluate the precision of the equation of state. Compared with the NIST data, the compressibility factor value obtained from the improved virial equation has a maximum error of $1.33 \%, 2.47 \%$ and $4.23 \%$ within the pressure ranges of $0.101325 \mathrm{MPa}<P<30 \mathrm{MPa}$ at the temperatures of $400 \mathrm{~K}, 300 \mathrm{~K}$ and $260 \mathrm{~K}$, respectively, and the precision of the improved virial equation of state is better than that of the existing P-R and S-R-K equations.

Also, the analytical expression for thermodynamic variables, such as the specific residual thermodynamic energy and specific residual enthalpy are presented to compensate the real gas effects, based on the improved virial equation of state. The study on dynamic thermodynamic analyses, mass flow rate, charging and discharging processes, and exergy analysis are of particular importance in high pressure air applications. In addition, based on the real thermodynamic variables, the internal ballistics mathematical model for a pneumatic ejection system is established, with the real gas effects considered. Numerical simulations are also performed.

The detailed dynamic thermodynamic processes for discharging processes in the hyperbaric chamber and charging processes in the low pressure chamber are analyzed. The comparison of the numerical results indicate that the value of residual enthalpy is high, the state of the working fluid deviates from the ideal gas, and the compressibility of working fluid is strong, as the compression factor reaches 1.107. The real gas effects accelerate the pressure and temperature rates of decrease in the hyperbaric pressure chamber, and decelerate the rates of increase in the low pressure chamber.

\section{Acknowledgments}

The authors acknowledge the support from the National Natural Science Foundation of China (Grant No. 51303081) and the Natural Science Foundation of Jiangsu Province, China (Grant No. BK20130761).

\section{Authors Contributions}

Dawei Ma conceived of the ideal that we can apply high pressure ejection device to weapon scope in line with the development direction for new concept weapons. His main contribution is the problem motivation. Jie Ren established the structure scheme of the high pressure pneumatic catapult, and developed the method to evaluate the real gas effects, by establishing compression factor library. Jie Ren also deduced the closed pneumatic equations to analyse the real dynamic thermodynamic process, by presenting the analytical expressions of specific residual thermodynamic energy and specific residual enthalpy of the high-pressure air based on real gas state equation. Fengbo Yang developed the research program to study the real gas effects, by using five-step four-order Runge-Kutta method to solve the closed pneumatic equations and calculated the compression factor based on P-R equation and improved virial equation. The contribution of Jianlin Zhong was to deduce the closed pneumatic equations based on ideal gas, including the mass and energy conservation laws. Guigao Le was responsible for the comparative analysis of the pneumatic catapult performance based on real gas state equation and ideal 
gas state equation, including the analysis of dynamic thermodynamic processes and article polish. All authors have read and approved the final manuscript.

\section{Nomenclature}

\begin{tabular}{|ll|ll|}
\hline$Z$ & compressibility factor, dimensionless & $h$ & specific enthalpy, $\mathrm{J} / \mathrm{kg}$ \\
$P$ & pressure, $\mathrm{MPa}$ & $R_{\mathrm{g}}$ & gas constant $\mathrm{J} /(\mathrm{kg} \cdot \mathrm{K})$ \\
$T$ & temperature, $\mathrm{K}$ & $s$ & specific entropy, $\mathrm{J} /(\mathrm{kg} \cdot \mathrm{K})$ \\
$V_{\mathrm{m}}$ & molar volume, $\mathrm{m}^{3} / \mathrm{mol}$ & $\alpha, \beta, \gamma, \delta, \varepsilon$ & constants \\
$R$ & universal gas constant, $\mathrm{J} /(\mathrm{mol} \cdot \mathrm{K})$ & $\rho$ & density, $\left(\mathrm{kg} / \mathrm{m}^{3}\right)$ \\
$B$ & the second virial cofficient, dimensionless & $n$ & polytropic exponent, dimensionless \\
$C$ & the third virial cofficient, dimensionless & Subscripts & \\
$\omega$ & acentric factor, dimensionless & $\mathrm{r}$ & corresponding value \\
$\Theta$ & extended corresponding states variables, dimensionless & $\mathrm{c}$ & critical value \\
$u$ & specific thermodynamic energy, $\mathrm{J} / \mathrm{kg}$ & $\mathrm{re}$ & residual value \\
$C_{\mathrm{v}}$ & isochoric heat capacity, $\mathrm{J} /(\mathrm{kg} \cdot \mathrm{K})$ & $*$ & ideal gas \\
$v$ & specific volume, $\mathrm{m}^{3} / \mathrm{kg}$ & 0 & reference state \\
\hline
\end{tabular}

\section{Conflict of Interest}

The authors declare no conflict of interest.

\section{References}

1. Luo, Y.; Wang, X. Exergy analysis on throttle reduction efficiency based on real gas equations. Energy 2010, 35, 181-187.

2. Zhu, J.; Lei, J.; Huang, Z.; Fu, T. Pneumatic position servo control system based on grey relational compensation control. Chin. J. Mech. Eng. 2012, 48, 159-167. (In Chinese)

3. Wang, X.; Luo, Y.; Xu, Z. Study of polytropic exponent based on high pressure switching expansion reduction. J. Therm. Sci. 2011, 5, 435-441.

4. Yang, J.L.; Ma, Y.T.; Li, M.X.; Guan, H.Q. Exergy analysis of transcritical carbon diodide refrigeration cycle with an expander. Energy 2005, 30, 1162-1175.

5. Chen, Z. Advanced Engineering Thermodynamics; Higher Education Press: Beijing, China, 2008.

6. Jia, G.; Wang, X.; Liu, H.; Wu, G. Study on pressure reduction and power property of high pressure pneumatic system on the compressed air powered vehicle. China Mech. Eng. 2004, 15, 1294-1297. (In Chinese)

7. Yang, G.; Guo, J.; Li, B. Dynamic simulation investigation of a novel high-pressure pneumatic proportional control valve. China Mech. Eng. 2007, 18, 1418-1420. (In Chinese)

8. Privat, R.; Privat, Y.; Jaubert, J.-N. Can cubic equations of state be recast in the virial form? Fluid Phase Equilibr. 2009, 282, 38-50.

9. Jaubert, J.-N.; Privat, R. Relationship between the binary interaction parameters $\left(\mathrm{k}_{\mathrm{ij}}\right)$ of the Peng-Robinson and those of the Soave-Redlich-Kwong equations of state: Application to the definition of the PR2SRK model. Fluid Phase Equilibr. 2010, 295, 26-37.

10. Scalise, O.H. On the phase equilibrium stockmayer fluids. Fluid Phase Equilibr. 2007, 253, 171-175. 
11. Brown, J.S. Predicting performance of refrigerants using the Peng-Robinson equation of state. Int. J. Refrig. 2007, 30, 1319-1328.

12. Avaullee, L.; Trassy, L.; Neau, E.; Jaubert, J. Thermodynamic modeling for petroleum fluids. I. Equation of state and group contribution for the estimation of thermodynamic parameters of heavy hydrocarbons. Fluid Phase Equilibr. 1997, 139, 155-170.

13. Privat, R.; Gani, R.; Jaubert, J.N. Are safe results obtained when the PC-SAFT equation of state is applied to ordinary pure chemicals? Fluid Phase Equilibr. 2010, 295, 76-92.

14. Bi, M.; Feng, D.; Ma, L. Engineering Thermodynamics; Chemical Industry Press: Beijing, China, 2008. (In Chinese)

15. Scott, D.J.; Patel, T.R.; Winzor, D.J. A Potential for overestimating the absolute magnitudes of second virial coefficients by small-angle X-ray scattering. Anal. Biochem. 2013, 435, 158-165.

16. Winzor, D.J.; Scott, D.J.; Wills, P.R. A simpler analysis for the measurement of second virial coefficients by self-interaction chromatography. Anal. Biochem. 2007, 371, 21-25.

17. Ramos-Estrada, M.; Iglesias-Silva, G.A.; Hall, K.R.; Kohler, F. Estimation of third virial coefficients at low reduced temperatures. Fluid Phase Equilibria. 2006, 240, 179-185.

18. Chen, H.; Zheng, J.; Xu, P.; Li, L.; Liu, Y.; Bie, H. Study on real-gas equations of high pressure hydrogen. Int. J. Hydrog. Energy 2010, 35, 3100-3104.

19. Xiang, H. Thermal Physical and Chemical Properties of the Fluid: The Corresponding State Principle and Application; Science Press: Beijing, China, 2003. (In Chinese)

20. Kameyama, H.; Yoshida, K.; Yamauchi, S.; Fueki, K. Evaluation of reference exergies for the elements. Appl. Energy 1982, 11, 69-83.

21. Lemmon, E.W.; Jacobsen, R.T.; Penoncello, S.G.; Friend, D.G. Thermodynamic properties of air and mixtures of nitrogen argon and oxygen from 60 to $2000 \mathrm{~K}$ at pressure to $2000 \mathrm{MPa}$. J. Phys. Chem. Ref. Data 2000, 29, 331-385.

22. Chueh, P.L.; Prausnitz, J.M. Third virial coefficients of nonpolar gases and their mixtures. AIChE J. 1967, 13, 896-902.

23. Meng, L.; Duan, Y.-Y.; Wang, X.-D. Binary interaction parameter kij for calculating the second cross-virial coefficients of mixtures. Fluid Phase Equilibr. 2007, 260, 354-358.

24. Meng, L.; Duan, Y.-Y.; Li, L. Correlations for second and third virial coefficients of pure fluids. Fluid Phase Equilibr. 2004, 226, 109-120.

25. Green, D.W.; Perry, R.H. Perry's Chemical Engineers' Handbook, 8th ed.; McGraw-Hill Professional: New York, NY, USA, 2007.

(C) 2014 by the authors; licensee MDPI, Basel, Switzerland. This article is an open access article distributed under the terms and conditions of the Creative Commons Attribution license (http://creativecommons.org/licenses/by/3.0/). 